Les sources de l'Histoire des Mines : Nouveaux outils, Nouvelles approches

Hélène Mialet, L'entreprise créatrice. Le rôle des récits, des objets et de l'acteur dans l'invention

Paris, Lavoisier - Hermes Science, 2008, 201 pages.

\title{
Michel Letté
}

\section{OpenEdition \\ Journals}

Édition électronique

URL : http://journals.openedition.org/dht/728

DOI : $10.4000 /$ dht. 728

ISSN : $1775-4194$

Éditeur :

Centre d'histoire des techniques et de l'environnement du Cnam (CDHTE-Cnam), Société des élèves du CDHTE-Cnam

\section{Édition imprimée}

Date de publication : 1 décembre 2008

Pagination : 222-225

ISBN : 978-2-95-30779-2-6

ISSN : 0417-8726

Référence électronique

Michel Letté, « Hélène Mialet, L'entreprise créatrice. Le rôle des récits, des objets et de l'acteur dans l'invention », Documents pour l'histoire des techniques [En ligne], 16। $2^{\mathrm{e}}$ semestre 2008, mis en ligne le 05 octobre 2010, consulté le 22 septembre 2020. URL : http://journals.openedition.org/dht/728 ; DOI https://doi.org/10.4000/dht.728

Ce document a été généré automatiquement le 22 septembre 2020

(c) Tous droits réservés 


\title{
Hélène Mialet, L'entreprise créatrice. Le rôle des récits, des objets et de l'acteur dans l'invention
}

\author{
Paris, Lavoisier - Hermes Science, 2008, 201 pages.
}

\section{Michel Letté}

\section{RÉFÉRENCE}

Hélène Mialet, L'entreprise créatrice. Le rôle des récits, des objets et de l'acteur dans l'invention, Paris, Lavoisier - Hermes Science, 2008, 201 pages.

1 Le titre aurait tout aussi bien pu comporter le rôle des procédures, celui de l'institution, des modèles, des pratiques ou bien des collègues et autres registres explorés dans ce livre afin d'y voir plus clair dans les modes d'existence de l'invention individuelle. Qu'est-ce que concevoir veut dire? Que mobilise-t-on dans ce travail particulier qui consiste à inventer? Ce sont quelques-unes des questions que l'auteure suggère de reconsidérer, invitant à aller au-delà de la distinction entre nature et social, à dépasser les clivages entre explication soit psychologisante, soit sociologisante de l'invention, et même à se méfier d'une dissolution trop rapide de la création individuelle dans les réseaux sociotechniques. Hélène Mialet s'intéresse aux modes de construction, d'attribution, d'appropriation, de distribution, de traduction, de validation, etc. ... et de façon générale à tout ce qui fait concrètement qu'une invention individuelle est validée comme telle par un collectif qui s'identifie en retour à elle, voire à l'individu lui-même dans le jeu d'une négociation mutuelle de sens.

2 L'intérêt porte sur une entreprise, et plus précisément sur un de ses laboratoires de recherches. L'unité de lieu est ici importante. Elle permet de comprendre et d'apprécier l'approche originale à laquelle conduisent les résultats de l'auteure qui ne sort pas de l'espace confiné du laboratoire. Elle respecte également les limites de la sphère individuelle de son « acteur-réseau ». Pas de visée ainsi sur le terrain de la psychologie 
individuelle ou de considérations d'ordre trop privé. On ne sait rien de plus de l'inventeur que ce qui est observé dans le cadre de l'exercice de sa profession. Les pratiques au quotidien sont scrutées, un point c'est tout. Si la question est ici traitée dans le cadre dédié d'une entreprise, l'auteure n'invite pour autant pas à généraliser ses conclusions à toute entreprise d'invention. C'est là un des points remarquable de sa démonstration qui incite alors à repenser en dehors du domaine restreint du laboratoire industriel nos intuitions les plus ancrées dans la certitude sur le statut et la nature de l'acte créatif.

3 François Montel est le héros de ce récit. Il est l'ingénieur-expert thermodynamicien des phases des fluides de gisements pétrolifères, qualifié par ses collègues et l'institution qui l'abrite d'inventeur d'une invention, devenue instrument pour la prise de décision opérationnelle sur les plateformes d'exploitation. Philosophe et sociologue, l'auteure nous entraîne ainsi sur son terrain d'étude anthropologique et ethnographique d'une tribu et de son chef qui exerce dans ce laboratoire de thermodynamique de l'entreprise Elf Aquitaine (depuis devenue Total), relié à l'économie monde de l'extraction du pétrole par une multitude d'écrans sur lesquels dansent des modélisations mathématiques de phases. Le chef de ce laboratoire est l'inventeur. L'inventeur reconnu est le chef légitime. Hélène Mialet a observé ce personnage et ses pratiques durant plusieurs années. Elle a élucidé ce qu'elle désigne, entre autres termes, par le «mécanisme inventif » mis en œuvre par François Montel afin d'entretenir, de cultiver, de maintenir cette catégorie « invention » comme catégorie opérante dans l'entreprise.

4 Pour permettre au lecteur de s'imprégner progressivement de la nature des enjeux de ce livre, l'auteure nous entraîne donc derrière ce chef de tribu où les bureaux équipés de moniteurs ont remplacé les paillasses, où le chef fait corps avec sa création, son invention. Si l'auteure prend la précaution de cultiver la distance avec son sujet, ses descriptions analytiques des pratiques du héros n'en sont pas moins restituées dans un style vivant, convenant pleinement à une étude de cette nature. Hélène Mialet donne à voir par exemple un François Montel quasiment en transe devant ses écrans, ses modèles mathématiques évoluant au gré du mouvement de ses doigts parcourant le clavier : «Ah je deviens diphasique, l'angoisse !», ainsi s'exprime l'inventeur embourbé dans sa mélasse pétrolifère en phase thermodynamique. Tribu étrange en vérité où les pratiques collectives semblent guidées par des rituels orchestrés par le chef. Il y a de toute façon quelque chose de magique dans ce qu'il fait, plus proche à certains égards des pratiques du sorcier que du chercheur de laboratoire. Il distille son savoir au fil des consultations et de la résolution des problèmes à l'évidence de façon très efficace, mais on ne comprend rien de l'excitation qui l'anime, de ce pourquoi il tape compulsivement sur les touches de son ordinateur et invoque dans des termes incompréhensibles des paramètres d'équation. Mais ça marche! François Montel est le grand chef, le sorcier des modèles thermodynamiques qui se diffusent dans des actions engagées sur les plateformes de gisement pétrolifères, mais sur lesquelles il ne met au demeurant jamais les pieds. Ceux qui l'entourent s'accordent pour dire qu'il est génial. L'institution en fait un modèle jusqu'à ce qu'il devienne lui même l'institution, et que l'institution s'incarne dans lui. Et peu importe s'il avoue au milieu de la démonstration en actes de ses compétences que ce qu'il a calculé la dernière fois est faux et que par conséquent ceux à qui il a vendu son étude ont dû louper quelques millions de tonnes (p. 39). Là n'est pas la question à l'évidence. C'est son invention et le fait qu'elle soit opérationnelle, qu'elle 
soit désignée comme telle, et qu'elle continue de l'être, notamment par la poursuite de rituels, qui est important.

Dès lors se repose la question de savoir comment la construction mutuelle de sens entre un existant technique, des savoirs associés, un individu, une entreprise deviennent, dans cet ensemble d'éléments hétérogènes, une invention à ce point reconnue par le collectif qu'il finit par l'incarner? L'étude des pratiques, de toutes les pratiques de François Montel comme acteur créatif tente d'y répondre. Hélène Mialet dote cependant son acteur créatif de nombreuses propriétés dont elle estime qu'elles étaient auparavant ignorées: celles de l'acteur «distribué-centré », notion essentielle développée dans ce livre, et qui prend toute sa consistance au fil de la lecture.

Pour en saisir pleinement les modes d'expressions, Hélène Mialet se penche sur le processus de singularisation de cet acteur-personnage, de son détachement du collectif en regard de ces compétences créatrices. Distingué par le collectif, François Montel contribue cependant lui même à le forger en s'y distribuant sous les formes les plus inattendues. D'abord sous la forme d'invention déclinée en modèles: modèles non seulement mathématiques devenus des instruments de la prise de décision opérationnelle, mais aussi de comportements, d'attitudes, de gestion des relations interpersonnelles et d'action. Pour le dire autrement, le processus de singularisation est l'objet véritable de l'étude proposée, et la notion d'acteur réseau distribué-centré la synthèse d'une proposition d'interprétation d'un mode d'existence de l'invention au sein de cette entreprise. François Montel constitue ainsi à la fois l'objet d'étude et la démonstration de devoir réintroduire le rôle individuel d'un acteur créatif comme principe explicatif d'une dynamique de l'innovation à la fois en actes et en discours, les uns et les autres devants se nourrir mutuellement pour maintenir leur légitimité et leur efficacité. Cet ouvrage se veut dès lors une réhabilitation de l'individu comme élément agissant de l'innovation. Il permet de réinvestir la question de l'acte créatif qui n'est plus celui du génie individuel consacré par la reconstruction de l'invention, mais celui de la construction d'un inventeur comme personnage distribué-centré.

7 L'invitation de l'auteure à concevoir la complexité de ces mécanismes pourra au premier abord susciter la perplexité. À force de changements de focale cependant, de déplacements du regard et d'allers et retours par d'autres voies sur des objets en perpétuel mouvement et pris dans des dynamiques de négociation de sens, on en vient à trouver des plus pertinents des concepts, des notions et des expressions qui pourtant ressembleraient à s'y méprendre à des oxymores. Derrière « l'individu collectif » ou "l'ontologie souple", les apparentes contradictions dans les termes sont en fait pleinement justifiées une fois que l'on a consenti à suivre Hélène Mialet. S'ouvre alors des environnements hétérogènes, des relations entre des individus, des collègues et des collaborateurs, des structures organisationnelles et hiérarchiques, des règles de fonctionnement explicites et tacites, des instruments, des critères d'évaluation, et en somme toute une série de techniques en actes qui font le devenir de l'inventeur et de l'invention. Autrement dit, c'est à une mise en configuration des interactions dynamiques entre des éléments hétérogènes (une phase instable et fugace dirait sans doute François Montel) à laquelle invite Hélène Mialet, où le sens, les fonctions et la nature même de l'acteur distribué-centré sont l'objet de tractations à nourrir en permanence. L'enjeu reste dans tous les cas de saisir la nature des liens entre ces acteurs, parfois hétérogènes, qui permettent à François Montel d'entretenir son mécanisme inventif. 
Hélène Mialet ne se contente cependant pas de reprendre en la complétant l'idée que l'invention est une construction humaine et sociale, et qu'à ce titre elle n'existe que par la médiation d'un processus de réalisation mutuelle de sens entre l'inventeur et le collectif dans lequel il s'insère. Certes elle enrichit à sa façon la pertinence des grilles d'interprétations s'appuyant sur les réseaux sociotechniques au travers desquels se négocient le devenir de l'innovation, mais elle supporte avec ce cas singulier un regard plus aigu permettant de renouer avec une forme de réhabilitation de l'individu et de l'individualité. Non pas sous couvert de replis sur la subjectivité, celle qui laisserait de côté les opérations complexes d'une construction négociée de réalité et de sens, par et pour le collectif, mais comme une investigation de l'espace pratique et contraint dans lequel l'individu opère, visant une reconnaissance de sa subjectivité pragmatique et de la rationalisation de sa pensée comme pratique de mise en adéquation de tensions entre des convictions individuelles et des prescriptions ou des injonctions pas nécessairement partagées ${ }^{1}$.

Hélène Mialet n'aborde cependant pas l'individu-acteur de front. Elle pratique en quelque sorte l'esquive du sujet créateur, non pour l'ignorer mais pour tirer de sa façon de faire des effets inattendus et produire de nouveaux matériaux d'analyse, de nouvelles observations. En quelque sorte, Hélène Mialet expérimente. Elle découvre, met au point elle-même une méthode, une démarche, une grille d'interprétation et de lecture des phénomènes qu'elle observe. Elle invente à son tour. C'est donc à une mise en abyme de l'auteure elle-même et de son travail à laquelle le lecteur assiste au fil de sa lecture, qui lui permet ainsi de partager d'une certaine façon son expérience. L'expérience pourrait sembler ne pas avoir de limites, car il y aurait matière à beaucoup commenter, à discuter, à débattre, à souhaiter voir d'autres pistes explorées dans cette quête des mécanismes inventifs dévoilés au travers des pratiques, soit en élargissant l'angle d'approche de tel objet, soit au contraire en resserrant la perspective.

10 Par exemple, la déconstruction de l'évidence du dispositif de distribution d'une prime à l'innovation dans l'entreprise est une démonstration convaincante de l'efficacité de ces jeux de focales opérés par l'auteure. Au travers de l'analyse des modalités d'attribution de cette prime dite de l'innovation, Hélène Mialet en vient ainsi à établir une fonction et un sens autres que ceux invoqués; à poser en fait la question de la nature implicite de ce que valident ces distributions de prix: des procédures de reconnaissance de compétences au sein du groupe certes, mais aussi un modèle institutionnel, une structure organisationnelle, l'autojustification d'existence d'un service dédié à l'innovation au titre d'entité opérationnelle autonome, des attitudes et des comportements individuels, des discours officiels, une culture d'entreprise.

11 La profondeur de vue est telle en tout cas que de nombreuses notes figurant en bas de page auraient mérité leur intégration au texte principal. Les analyses sont denses, très denses, mais fort heureusement souvent et autrement répétées. Hélène Mialet revient sur ses développements antérieurs, pour les enrichir, les affecter d'un regard ou d'un point de vue décalé. Il ne faudrait cependant pas croire que l'ouvrage ne comporterait qu'une dimension analytique. Exercice et étape incontournables en début d'ouvrage, Hélène Mialet rappelle ainsi que la question du «comment » et du possible même de l'invention n'a jamais été simple et ne peut l'être. Elle y expose ainsi dans une synthèse remarquable l'éventail des approches proposées par les philosophes et les sociologues des sciences de ce qu'est l'idée d'invention comme idée neuve, s'imposant aux acteurs 
sociaux et économiques d'une façon ou d'une autre comme une évidence, et dont il ne resterait finalement qu'à élucider le mécanisme naturel d'émergence, soit à l'esprit individuel du "génie génial sachant innover », soit déterminé par des facteurs sociaux au sein d'une société toujours plus avide de progrès techniques. La plupart des philosophes, mais aussi des sociologues des sciences, a de fait eu le souci d'évacuer la question de la création elle-même, c'est-à-dire de celle de l'acte créatif. C'est à partir de ce chantier trop vite clôturé que l'auteure a réinvesti la question de la créativité individuelle, non pas en soi, dans sa dimension ontologique, mais dans le cadre d'une phénoménologie délimitée par les frontières institutionnelles d'une entreprise, c'est-àdire en décrivant d'abord ce que les acteurs font, disent et comment ils voient le monde immédiat qu'ils contribuent à faire exister.

12 La fin de l'ouvrage arrive vite, trop vite, et on sera peut être frustré, par exemple au détour de la seule évocation dans ce cadre particulier de production de l'invention de la dimension performative des énoncés produits par l'institution et les actes individuels qu'ils induisent. À l'issue de la lecture, l'invention n'est cependant pas plus un donné stable qu'au début de l'ouvrage. Elle n'est toujours pas le fruit d'une découverte ou d'un trait fulgurant fixé une fois pour toute. L'invention, ou tout au moins ce qui est qualifié comme tel, s'est au contraire consolidée comme processus dynamique de construction mutuelle, qui a besoin d'être entretenu, de voir se maintenir son statut particulier pour continuer à assurer ses fonctions au sein de l'entreprise. Pas de génie créateur donc mais seulement un génie individuel. L'acte de création inventive reste plein de conditions sociales et économiques qui président à la possibilité de son émergence pour l'existence. L'inventeur ne disparaît pas. Il prend au contraire de l'épaisseur dans sa fonction d'acteur distribué-centré d'un réseau sociotechnique.

Toutes ces questions peuvent à certains égards sembler ne pas être tout à fait neuves dans une entreprise, mais les propositions de lecture pertinentes auxquelles aboutit Hélène Mialet commandent assurément de conserver à portée de main cet ouvrage, à relire dès que l'on sera confronté à la complexité des dispositifs qui consistent à faire exister dans le réel et advenir comme réalité sociale l'invention. Au fait, de quelle invention au juste était-il question dans ce livre remarquable?

\section{NOTES}

1. Sur cette question de la place de la subjectivité dans les processus collectifs de production technique, on verra l'article d'Yves Cohen paru dans le dernier numéro de la revue : «L'ingénieur et sa pratique. Les techniques et la subjectivité », Documents pour l'histoire des techniques, 15, 2008, pp. 77-89. 


\section{AUTEURS}

MICHEL LETTÉ

CDHTE-Cnam 